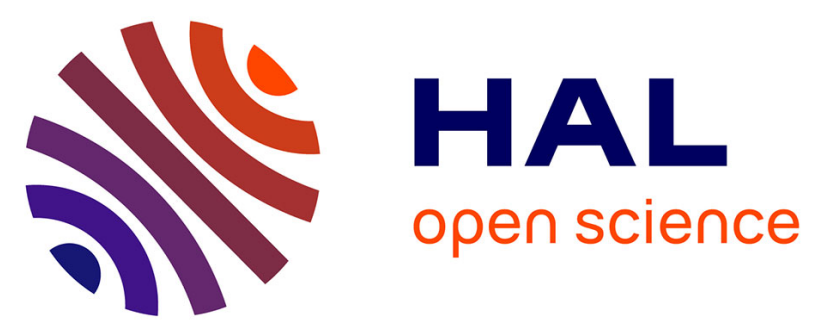

\title{
Biochemical and functional characterization of Rab27a mutations occurring in Griscelli syndrome patients
}

Gaël Menasche, Jerôme Feldmann, Anne Houdusse, Catherine Desaymard, Alain Fischer, Bruno Goud, Geneviève de Saint Basile

\section{- To cite this version:}

Gaël Menasche, Jerôme Feldmann, Anne Houdusse, Catherine Desaymard, Alain Fischer, et al.. Biochemical and functional characterization of Rab27a mutations occurring in Griscelli syndrome patients. Blood, 2003, 101, pp.2736 - 2742. 10.1182/blood-2002-09-2789 . hal-03059374

\section{HAL Id: hal-03059374 https://hal.science/hal-03059374}

Submitted on 12 Dec 2020

HAL is a multi-disciplinary open access archive for the deposit and dissemination of scientific research documents, whether they are published or not. The documents may come from teaching and research institutions in France or abroad, or from public or private research centers.
L'archive ouverte pluridisciplinaire HAL, est destinée au dépôt et à la diffusion de documents scientifiques de niveau recherche, publiés ou non, émanant des établissements d'enseignement et de recherche français ou étrangers, des laboratoires publics ou privés. 


\title{
Biochemical and functional characterization of Rab27a mutations occurring in Griscelli syndrome patients
}

\author{
Gaël Ménasché, Jérôme Feldmann, Anne Houdusse, Catherine Desaymard, Alain Fischer, Bruno Goud, and Geneviève de Saint Basile
}

Rab27a is a member of the Rab family of small GTPase proteins, and thus far is the first member to be associated with a human disease (ie, the Griscelli syndrome type 2). Mutations in the Rab27a gene cause pigment as well as cytotoxic granule transport defects, accounting for the partial albinism and severe immune disorder characteristics of this syndrome. So far, 3 Rab27a missense mutations have been identified. They open a unique opportunity to designate critical structural and functional residues of Rab proteins. We show here that the introduction of a proline residue in the $\alpha 4$ (Ala152Pro) or $\beta 5$ (Leu130Pro) loop, observed in 2 of these spontaneous mutants, dramatically affects both guanosine triphosphate (GTP) and guanosine diphosphate (GDP) nucleotide-binding activity of Rab27a, probably by disrupting protein folding. The third mutant, Trp73Gly, is located within an invariant hydrophobic triad at the switch interface, and was previously shown in active Rab3A to mediate rabphilin3A effector interaction. Trp73Gly is shown to display the same nucleotidebinding and GTPase characteristics as the constitutively active mutant GIn78Leu. However, in contrast to GIn78Leu, Trp73Gly mu- tant construct neither interacts with the Rab27a effector melanophilin nor modifies melanosome distribution and cytotoxic granule exocytosis. Substitutions introduced at the 73 position, including the leucine residue present in Ras, did not restore Rab27a protein functions. Taken together, our results characterize new critical residues of Rab proteins, and identify the Trp73 residue of Rab27a as a key position for interaction with the specific effectors of Rab27a, both in melanocytes and cytotoxic cells. (Blood. 2003;101:2736-2742)

(c) 2003 by The American Society of Hematology

\section{Introduction}

The Rab family of low-molecular-weight GTPases plays an essential role in intracellular transport vesicles by controlling membrane trafficking between intracellular compartments. Rab GTPases interact specifically with a subset of effector proteins that, in turn could impart specificity to membrane trafficking at the levels of vesicle translocation, docking, and fusion. ${ }^{1-3}$ In mammalian cells, more than 50 Rab proteins have now been identified. ${ }^{4-6}$ Rab27a is the only one so far that has been associated with a human disease (ie, the Griscelli syndrome). We previously reported that the Griscelli syndrome, characterized by a partial albinism of the skin and hair and a severe immunologic disorder, results from Rab27a deleterious mutations. ${ }^{7}$ Although Rab27a is wildly expressed, the clinical phenotype of the patients with Griscelli syndrome indicates a determinant role of this Rab protein in melanocytes and lymphocytes. In addition to its role in melanosome peripheral transfer, we have shown that Rab27a is required for cytotoxic granule exocytosis, and that the defective cytotoxic activity of patients' lymphocytes resulting from Rab27a mutation accounts for the severe immune disorder characteristic of this syndrome, known as hemophagocytic syndrome (HS). ${ }^{7,8} \mathrm{HS}$ is characterized by lymphoid organ and extranodal infiltration by polyclonal activated $\mathrm{T}$ cells, mostly of the $\mathrm{CD}^{+}$subset, and by activated macrophages that phagocytose blood cells. This condi- tion does not, in most cases, spontaneously remit. Immune cell infiltration results in massive tissue necrosis, organ failure, and death in the absence of immunosuppressive treatment. Massive cytokine release is another hallmark of the condition, and it is thought that macrophage activation is mostly the consequence of tumor necrosis factor (TNF) production, leading to hemophagocytosis, high triglyceride levels, and coagulation disorders. Similarly, in the ashen mice, Rab27a mutation leads to defects in melanosome transport and granule exocytosis in cytotoxic $\mathrm{T}$ lymphocytes. ${ }^{9-11}$ Recently, the product of the gene mutated in the leaden $\left(\mathrm{Mlph}^{d}\right)$ mice, ${ }^{12}$ the melanophilin (Mlph; also known as Slac2-a), was identified as an effector of Rab27a in melanocytes. ${ }^{12-15}$ The Mlph bridges Rab27a and the molecular motor myosin $\mathrm{Va}$, the tripartite protein complex being required for melanosome transport function. ${ }^{16,17}$

The molecular mechanisms, which underlie the diversity of Rab/effector interaction, are not yet fully understood. Rabs cycle between a guanosine triphosphate (GTP)-bound (active) and a guanosine diphosphate (GDP)-bound (inactive) conformation, but this highly conserved mechanism among GTPase proteins cannot account for the discrimination of diverse effectors and regulatory factors. In addition to Rab-specific motifs present in all Rab GTPase, Rab subfamily-specific sequences (RabsF), with high-
From the Unité de Recherche sur le Développement Normal et Pathologique du Système Immunitaire Institut National de la Santé et de la Recherche Médicale (INSERM) U429, Hôpital Necker-Enfants Malades, Paris, France; and équipe Motilité structurale and équipe Mécanismes moléculaires du transport intracellulaire, Unité Mixte de Recherche, Institut Curie/Centre National de la Recherche Scientifique 144, Institut Curie, Paris, France.

Submitted September 13, 2002; accepted November 12, 2002 Prepublished online as Blood First Edition Paper, November 21, 2002; DOI 10.1182/blood-2002-09-2789.

Supported by grants from INSERM, association de Recherche sur le Cancer
(ARC 5849; G.de S.B. and B.G.), and association Vaincre les Maladies Lysosomales (VML).

Reprints: Geneviève de Saint Basile; INSERM U 429, Hôpital Necker-Enfants Malades, 149 rue de Sèvres, Paris Cedex 15, 75743 France; e-mail: sbasile@necker.fr.

The publication costs of this article were defrayed in part by page charge payment. Therefore, and solely to indicate this fact, this article is hereby marked "advertisement" in accordance with 18 U.S.C. section 1734.

(C) 2003 by The American Society of Hematology 
amino-acid conservation within subfamilies, have been identified and are thought to participate in the specificity of regulator interaction. ${ }^{5}$ Crystallographic studies of the constitutively active mutant of Rab3A and its effector, rabphilin3A, have defined 3 hypervariable Rab complementary-determining regions (Rab CDRs) involved in molecular interaction. They appear as major determinants of functional effector specificity. ${ }^{18}$ Interestingly, all 3 Rab CDRs are included in the subfamily motifs defined by sequence homology analysis. ${ }^{5}$ An invariant hydrophobic triad at the switch interface of active Rab3A was shown to mediate central interaction with rabphilin3A. ${ }^{18}$ Spatial exposition of this triad in the active form of Rab5C is noncomplementary to the switch interaction epitope of rabphilin3A, supporting the specific contribution of these hydrophobic residues in effector recognition. ${ }^{19}$ Rab3A and Rab27a are phylogenetically similar and share $43 \%$ identity of amino acid residues. Several effectors of Rab27a have been described either belonging to the synaptotagmin-like protein (Slp) family (Slp1/JFC1, Slp2, Slp3, Slp4/granuphilin, and Slp5) or to the Slac2 (Slp homologues lacking C2 domain) family (Slac2/ melanophilin, Slac2b, and Slac2c/MyRIP). All of them were shown to interact with Rab27a through a conserved N-terminal Slp homology domain (SHD), which was identified by Kuroda et al. ${ }^{14}$ The Rab effector domain of rabphilin-3A and the SHD domain of melanophilin are conserved and share $26 \%$ identity and $43 \%$ similarity. Therefore, a closely similar structural interaction is likely to occur as confirmed by the ability of rabphilin-3A to specifically interact in vitro with Rab27a. ${ }^{14}$

Most Rab27a mutations so far identified in Griscelli syndrome patients are null mutations resulting from nonsense mutations or frameshift deletions (Ménasché et $\mathrm{al}^{7}$; and G.d.S.B., personal data, October 2002). However, we here identified 3 inherited homozygous missense mutations in the Rab27a gene (Trp73Gly, Leu130Pro, and Ala152Pro) that are associated with Griscelli syndrome. ${ }^{7}$ These spontaneous mutants represent a unique opportunity to evaluate how these designated critical residues of Rab27a, and more broadly, perhaps, of Rab proteins, may participate in Rab protein functions. We thus analyzed nucleotide-binding activity and Mlphbinding ability, as well as the potential transdominant effect of these various mutants expressed in melanocytes and cytotoxic cells. We report that the insertion of a proline residue either in the $\alpha 4$ or $\beta 5$ loop found in 2 of these mutants dramatically impairs both GTP and GDP nucleotide-binding, probably by disrupting protein folding. The third mutant, characterized by one residue substitution in the invariant hydrophobic triad, ${ }^{19}$ was shown to exhibit a similar nucleotide binding-site activity compared with the constitutively active mutant Gln78Leu, but differs significantly in effector interaction and functional properties when overexpressed in cells. These data strongly support the physiologic relevance of the invariant hydrophobic triad in effector recognition and suggest that the specific effectors of Rab27a in both melanocytes and lymphocytes require similar structural constraints for Rab27a interaction.

\section{Patients, materials, and methods}

\section{Patients}

In the Rab27a gene, 3 missense mutations (Trp73Gly, Leu130Pro, and Ala152Pro) were identified among 38 patients with Rab27a- Griscelli syndrome type 2 (G.d.S.B., personal data, October 2002). Mutations in 3 of these patients (P9, P15, and P17) have been previously reported. ${ }^{7,20}$ In a fourth patient (P37), the same homozygous T217G mutation leading to Trp73Gly transition as in P9 was found. P9 and P37 are both from Turkish origin and their mutation may thus originate from a common ancestor. All 4 patients exhibited the characteristic phenotypic features of Griscelli syndrome $^{21}$ (ie, association of silvery-gray sheen of the hair with large pigment aggregations in hair shafts and an immune defect characterized by the occurrence of at least one episode of HS). P15 (with Leu130 mutation) presented with a severe form of the disease, as observed in most of the patients with a null mutation in the Rab27a gene, with the occurrence at the age of 2.5 months of a hemophagocytic syndrome, which was rapidly fatal. P9 (Trp73Gly), P17 (Ala152Pro), and P37 (Trp73Gly) had a relatively late onset of the first hemophagocytic syndrome episode (ie, 8 years for P9, and 4 years for both P17 and P37). ${ }^{20}$

\section{Molecular cloning of human Rab27a cDNA and mutants}

Wild-type (WT) Rab27a cloning. A cDNA encoding a full open-reading frame of Rab27a was obtained by reverse transcription-polymerase chain reaction (RT-PCR), using SuperScript One-Step RT-PCR System with platinum Taq (Invitrogen, Cergy Pontoise, France) from total RNA of human B-EBV lymphoblastoid cell line as described previously. The following primers were used: EcoRI-Rab27a-Met-sense, 5'-GGAATTCCATGTCTGATGGAGATTATGA-3' and BamHI-Rab27a-stop-antisense, 5'GCGGATCCGCCCACCTGAACTACTATGTCA-3'. Sequence site recognition of the corresponding enzymes are underlined. Digested PCR product was cloned into pcDNA 3.1 (Invitrogen), pFlag-CMV-4 (Sigma, Saint Quentin Fallavier, France), and pEGFP-C1 (Clontech, Basingstoke, United Kingdom). For in-frame cloning with His6-tag in P15b, ${ }^{22}$ the following primers were used to generate the PCR product: SmaI-Rab27a-Met, 5'-TCCCCCGGGGATATGTCTGATGGAGATTATGA-3' sense primer; and BamHI-Rab27a-stop antisense primer.

Mutants Rab27a cloning. Site-directed mutagenesis (in bold on the sequences) of Rab27a was performed using the double-PCR strategy. ${ }^{23}$ The primers used are as follows: 5'-TGTAGGGAAGAACAGTGT-3' (Thr23Asn primer; sense); 5'-TGGTAAAGTACACTGTTCTT-3' (Thr23Asn primer; antisense); 5'-GCAGTTAGGGGACAC-3' (Trp73Gly primer; sense); 5'-GTCCCCTAACTGCAGGTGGATT-3' (Trp73Gly primer; antisense); 5'-GCAGTTACTGGACAC-3' (Trp73Leu primer; sense); 5'-GTCCAGTAACTGCAGGTGGATT-3' (Trp73Leu primer; antisense); 5'-GCAGTTATCGGACAC-3' (Trp73Ser primer; sense); 5'-GTCCGATAACTGCAGGTGGATT-3' (Trp73Ser primer; antisense); 5'-GGCTGGAGAGGTTTCGT-3' (Gln78Leu primer; sense); 5'-CTCCAGCCCTGCTGTG-3' (Gln78Leu primer; antisense); 5'-AGTGCCGTGTGGAAACAA-3' (Leu130Pro primer; sense); 5'-TTGTTTCCACACGGCACT-3' (Leu130Pro primer; antisense); 5'-ATAGCACTCCCAGAGAAA-3' (Ala152Pro primer; sense); and 5'-TTTCTCTGGGAGTGCTAT-3' (Ala152Pro primer; antisense). The amplified PCR products were digested by SmaI and BamHI and subcloned into pcDNA 3.1 Pet15b/His and pFlag-CMV-4 and pEGFP-C1 vectors.

\section{Molecular cloning of the SHD domain of human Mlph}

A cDNA encoding the first 146 amino acids of human Mlph, termed SHD, ${ }^{14}$ was synthesized from total RNA isolated from the human melanoma cell line MNT-1 (gift from P. G. Natali, Instituto Regina Elena, Rome, Italy) by one-step RT-PCR with the following primers: 5'-GGAATTCATGGGGAAGAAACTGGAT-3' (EcoRI-Mlph-Met primer; sense) and 5' -CTCGAGCTGCATCCGCCCGTGGAGGGACCG-3' (XhoI-Mlph 146 primer; antisense; sequence site recognition of the corresponding enzymes are underlined.). The digested PCR product was cloned in frame into pcDNA 3.1/Myc-His (Invitrogen), which adds the Myc epitope at the $\mathrm{C}$ terminus of the cloned cDNA.

\section{Purification of recombinant proteins}

Escherichia coli BL21 cells were transformed with recombinant plasmid Pet $15 \mathrm{~b}$ containing the correct inserts and grown in Luria-Bertani (LB) medium with $30 \mu \mathrm{g} / \mathrm{mL}$ ampicilin. When optical density $\left(\mathrm{OD}_{600}\right)$ was 0.6 , isopropyl-1-thio- $\beta$-D galactopyranoside was added to a final concentration of $1 \mathrm{mM}$. Cells were harvested 3 hours later by centrifugation at $1500 \mathrm{~g}$ for 10 minutes. Bacterial pellets were resuspended in $10 \mathrm{mM}$ Tris- $\mathrm{HCl}, \mathrm{pH} 8.8$; $300 \mathrm{mM} \mathrm{NaCl} ; 1 \mathrm{mM}$ dithiothreitol (DTT); $10 \mathrm{mM}$ imidazol; $10 \mu \mathrm{M}$ GTP; 
and $10 \mu \mathrm{g} / \mathrm{mL}$ phenylmethylsulfonyl fluoride (PMSF), and sonicated at $4^{\circ} \mathrm{C}$. The lysate was centrifuged twice at $10000 \mathrm{~g}$ for 10 minutes. The mutants Leu130Pro and Ala152Pro were solubilized in $0.8 \%$ sodium dodecyl sulfate (SDS), $250 \mu \mathrm{M}$ triethanolamine, $1.5 \%$ sarcosyl, and $0.5 \mathrm{M}$ EDTA (ethylenediaminetetraacetic acid). Purification was carried out according to the instruction manual of the QIAexpressionist (Qiagen SA, Courtaboeuf, France). Briefly, the supernatant was incubated with $300 \mu \mathrm{L}$ nickel-nitrilo tri-acetic acid (Ni-NTA) resin and mixed gently for 2 hours at $4^{\circ} \mathrm{C}$. The recombinant proteins were washed and eluted with $250 \mathrm{mM}$ imidazol. Before the binding assays, the proteins were dialyzed overnight against $20 \mathrm{mM}$ Tris- $\mathrm{HCl}, \mathrm{pH} 7.5 ; 1 \mathrm{mM}$ DTT; and $5 \mathrm{mM} \mathrm{MgCl}_{2}$. The purity was estimated by SDS-polyacrylamide gel electrophoresis (PAGE) and coomassie blue staining. Protein concentration was determined by a Bradford assay (Pierce-Perbio Science, Bezons, France) using bovine serum albumin (Sigma) as a standard.

\section{Binding of nucleotides}

Recombinant proteins were incubated in $20 \mathrm{mM}$ Tris- $\mathrm{HCl}, \mathrm{pH} 7.5 ; 1 \mathrm{mM}$ DTT; $5 \mathrm{mM} \mathrm{MgCl} 2 ; 10 \mathrm{mM}$ EDTA; $0.5 \mathrm{~g} / \mathrm{L}$ bovine serum albumin; $\left({ }^{3} \mathrm{H}\right) \mathrm{GTP}$ or $\left({ }^{3} \mathrm{H}\right) \mathrm{GDP}(7.7 \mathrm{Ci} / \mathrm{mmol}[284.9 \mathrm{GBq} / \mathrm{mmol}]$, Amersham Pharmacia Biotech, Saclay, France); and cold $30 \mu \mathrm{M}$ GTP or GDP. After incubation at $30^{\circ} \mathrm{C}$ for the indicated times, samples were diluted in $500 \mu \mathrm{L}$ ice-cold washing buffer (20 mM Tris- $\mathrm{HCl}, \mathrm{pH} 7.5 ; 25 \mathrm{mM} \mathrm{MgCl}$; and $100 \mathrm{mM}$ $\mathrm{NaCl})$ and applied to a nitrocellulose filter $(0.45 \mu \mathrm{m}$; Millipore, Saint Quentin en Yvelines, France). The filters were rinsed with $4 \times 4 \mathrm{~mL}$ ice-cold washing buffer, and the radioactivity retained on the filters was determined by scintillation counting. ${ }^{24}$

\section{GTPase activity}

In the condition described above, proteins were preincubated for 1 hour for nucleotide binding with $\left(\gamma_{-}{ }^{32} \mathrm{P}\right) \mathrm{GTP}(5000 \mathrm{Ci} / \mathrm{mmol}[185000 \mathrm{GBq} / \mathrm{mmol}]$, Amersham Pharmacia Biotech), and then $20 \mathrm{mM} \mathrm{MgCl}_{2}$ was added to the final concentration. At the indicated times, samples were diluted with cold buffer, filtered, and counted.

\section{Cell culture, transient transfection, and functional assay}

The immortal control-mouse melanocyte cell line, melan-a, was kindly provided by Dorothy Bennett, (St George's Hospital Medical School, London, United Kingdom). Melan-a was cultured in RPMI 1640 supplemented with $10 \%$ fetal calf serum (FCS) and $200 \mathrm{nM}$ phorbol myristate acetate (PMA; Sigma) at $37^{\circ} \mathrm{C}$ with $10 \% \mathrm{CO}_{2}$. For microscopic analysis, cells were grown on coverslips for 24 hours and cotransfected using the liposomal transfection reagent Fugene 6 (Roche Diagnosis, Meylan, France) with $2.5 \mu \mathrm{g}$ of pEGFP-C1 (Clontech), allowing soluble green fluorescence protein (GFP) expression, and $2.5 \mu \mathrm{g}$ of pcDNA containing Rab27a WT or mutants and/or Mlph. Cells were fixed 24 hours later in 3.7\% paraformaldehyde for 15 minutes, washed extensively, and mounted in a medium containing Mowiol antifading agent (Calbiochem, San Diego, CA). Cells were observed using an Axioplan 2 microscope (Zeiss, Jena, Germany). The 293 T cell line ${ }^{25}$ was cultured in Dulbecco modified Eagle medium (DMEM) with $10 \% \mathrm{FCS}$ at $37^{\circ} \mathrm{C}$ with $5 \% \mathrm{CO}_{2}$ and transfected by electroporation (250 V, $900 \mu \mathrm{F}$ [EquiBio, Ashford, United Kingdom]). Herpesvirus saimiri-transformed $\mathrm{CD} 8^{+} \mathrm{T}$ cells ${ }^{26}$ were kept continuously in medium containing interleukin-2 (IL-2; $40 \mathrm{U} / \mathrm{mL}$ ). This T-cell line was transiently transfected with various EGFP-Rab27a constructs using the Human T-cell Nucleofector Kit (Amaxa Biosytem, Köln, Germany), and degranulation assay was tested 18 hours later using N-a-benzyloxycarbonylL-lysine thiogbenzyl ester (BLT) esterase release analysis. Intracellular expression of the various constructs was evaluated by FACScan analysis (Becton Dickinson, San Diego, CA). Peripheral blood lymphocytes from patients or controls were stimulated with phytohemagglutinin (PHA, 1:700 dilution; Difco, Detroit, MI) for 24 hours and then cultured in IL-2 (40 $\mathrm{U} / \mathrm{mL}$; Valbiotech, Paris, France) for 6 days. The degranulation assay was done on these cells as previously described, ${ }^{7}$ with some modifications. Briefly, anti-CD3 antibody ( $20 \mu \mathrm{g} / \mathrm{mL}$; OKT3, Ortoclone; Jansen, France) coated in 96-well plates was used instead of target cells to stimulate $6 \times 10^{5} \mathrm{~T}$ cells/well or
$2.5 \times 10^{5}$ transfected $\mathrm{H}$ saimiri $\mathrm{CD} 8^{+} \mathrm{T}$ cells/well in a 4-hour release assay. The release of BLT esterase activity on a $50-\mu \mathrm{L}$ aliquot of cell-free supernatant was performed as previously described.

\section{Immunoprecipitation}

Flag-Rab27a WT or mutants and ${ }_{1-146} \mathrm{Mlph}-$ Myc were coexpressed in $293 \mathrm{~T}$ cells. One day after electroporation, cells were lysed in $50 \mathrm{mM}$ Tris, $\mathrm{pH} 8.0$; $1 \%$ nonidet P-40; and $2 \mathrm{mM}$ EDTA, supplemented with $10 \mu \mathrm{g} / \mathrm{mL}$ each of protease inhibitors leupeptin, aprotinin, N-tosyl-L-phenylalanine chloromethyl ketone, N-p-tosyl-L-lysine chloromethyl ketone, and PMSF, as well as the phosphatase inhibitors sodium fluoride $(50 \mathrm{mM})$ and sodium orthovanadate $(1 \mathrm{mM}){ }^{27}$ Anti-c-Myc (9E10; Santa Cruz Biotechnology, Hedelberg, Germany) and anti-Flag-M2 (Sigma) were used for immunoprecipitation. Immune complexes were collected with protein $\mathrm{G}$ (Sigma). Proteins were loaded in 12\% SDS-PAGE and transferred onto Immobilon membrane (Millipore).

\section{Results}

\section{Nucleotide binding and GTPase activity of Rab27a mutants}

There were 3 different homozygous missense mutations of the Rab27a gene found to be associated with Griscelli syndrome (Leu130Pro, Ala152Pro, and Trp73Gly), pointing out the biologic relevance of these amino acid transitions. Based on the crystal structure of GTP-Rab3A, ${ }^{18}$ Leu130Pro is located on the $\beta 5$ sheet, Ala152Pro on the $\alpha 4$ loop, and Trp73Gly on the $\beta 3$ sheet near the nucleotide-binding site (Figure 1). To evaluate the molecular consequences of these substitutions, the Rab27a mutants were expressed and tested for their nucleotide-binding ability as well as GTPase activity. In addition, the constitutively GTP-bound, active Rab27a Gln78Leu mutant (equivalent to Rab3A Gln81Leu in Figure 1), as well as the Rab27a Thr23Asn mutant (corresponding to Rab3A Thr36Asn in Figure 1), which is predominantly in the GDP-bound, inactive conformation, were also constructed and tested. As expected, WT Rab27a and Gln78Leu mutant bind to GTP (Figure 2A). Mutant Trp73Gly was shown to exhibit a similar GTP-binding ability, although with lower kinetics. These 3 proteins also bind to GDP as does the Thr23Asn mutant (Figure 2B). In contrast, Leu130Pro and Ala152Pro mutants did not bind to GTP or GDP nucleotides (Figure 2A-B). The introduction of a proline at the mutation sites most likely impairs the folding of these proteins as suggested by the specific detergent conditions required for their

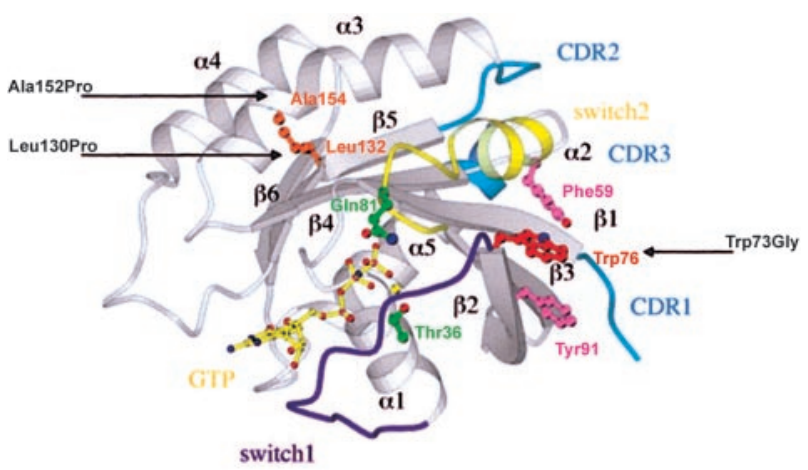

Figure 1. Localization of Rab27a mutations on Rab3A structure. Ribbon representation of the Rab3A/GTP-bound structure with the putative switch regions highlighted in purple (switch 1) and yellow (switch 2), and the CDRs in blue. The amino acids mutated in Rab27a- patients (Leu130Pro, Ala152Pro, and Trp73Gly) are indicated with an arrow at their equivalent positions on the Rab3A structure, Leu132 and Ala154 (in brown) and Trp76 (in red). The constitutively active (GIn78Leu) and inactive (Thr23Asn) Rab27a mutations are shown in green at their corresponding Rab3A residues, Gln81 and Thr36, respectively. Phe59 and Tyr91 (in pink) and Trp76 (in red) define the invariant hydrophobic triad. 
A
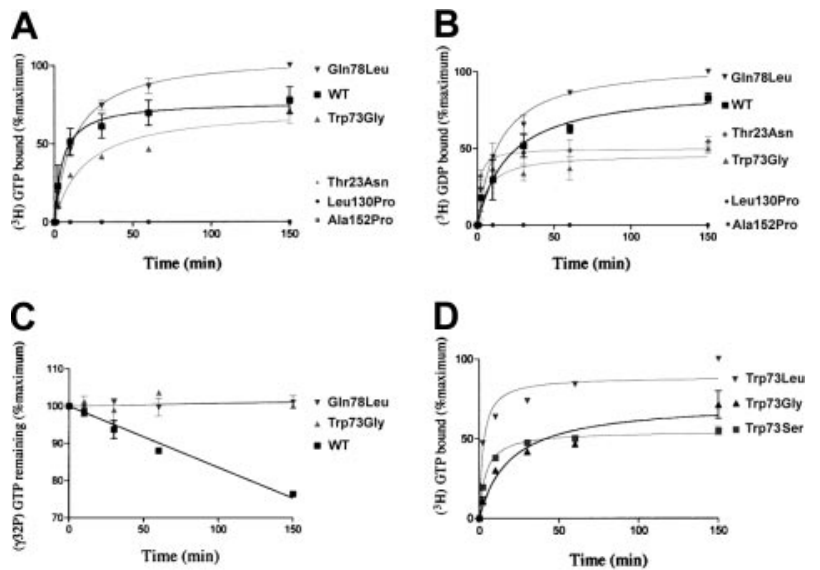

E

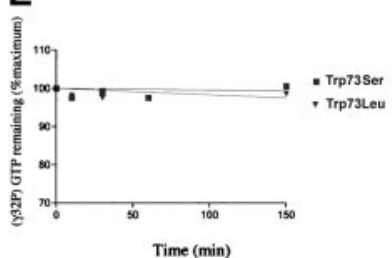

Figure 2. Nucleotide-binding rates and GTPase activities of Rab27a and the various mutants. Nucleotide-binding and GTPase activity rates were measured by the filtration method, and the data are expressed as the percentage of the maximum radioactive nucleotide binding to Rab27a WT or mutant proteins. (A) GTP binding The Rab27a (WT) and mutant (Thr23Asn, Trp73Gly, Leu130Pro, and Ala152Pro) proteins were incubated with $\left({ }^{3} \mathrm{H}\right)$-GTP for the indicated times, and the amount of bound $\left({ }^{3} \mathrm{H}\right)$-GTP was determined. (B) GDP binding. Fixation of $\left({ }^{3} \mathrm{H}\right)$-GDP with the same recombinant proteins. (C) GTPase activities of proteins WT (ש), Trp73Gly (gray triangle), and GIn78Leu (inverted gray triangle). The WT and mutant Rab27a proteins were loaded with $\left(\gamma^{-32}\right.$ P)GTP, and GDP hydrolysis was determined by the release of $\left.{ }^{(32} \mathrm{P}\right) \mathrm{Pi}$ (phosphate inorganic). (D) GTP binding. Fixation of $\left({ }^{3} \mathrm{H}\right)$-GTP with 2 new mutations at position 73: Trp73Ser, Trp73Leu as performed in panel A. (E) GTPase activities of proteins Trp73Ser (gray square) and Trp73Leu (inverted gray triangle) performed in the same conditions as in panel $C$. The results show 3 different experiences performed in duplicates and analyzed by nonlinear regression with the program Prism 3 (Graph Pad Software, San Diego, CA).

purification. Since both GTP- and GDP-binding activity were maintained when WT was prepared in the same conditions (data not shown), defective binding activity appears strictly related to the presence of the proline and not to the experimental procedure used.

The GTPase activity of the Rab27a proteins was next evaluated. Only the WT form of Rab27a had an intrinsic GTP hydrolysis activity, whereas Trp73Gly and Gln78Leu were inefficient in hydrolyzing GTP (Figure 2C). By analogy to other small GTPase proteins, the conserved Gln78 residue is expected to stabilize the catalytic transition state between switch I and II. Transition to leucine may lower this catalytic rate and lock the Rab protein into the activated, GTP-bound state. Residue 73 (residue 76 of Rab3A in Figure 1) is located in the $\beta 3$ sheet at the interface between the 2 switch regions. Similarly, its mutation alters GTPase activity, possibly by modifying the conformational change of the P-Loop or the switch regions. We hypothesized that a Trp $\rightarrow$ Gly transition may have increased switch flexibility and thus impaired the dynamics of the conformational changes associated with each nucleotide binding. To test this hypothesis, 2 other mutations at position 73 were generated: either a leucine residue (Trp73Leu), as in the p21 Ras protein, or a serine residue (Trp73Ser), which is supposed to limit switch II conformational liability possibly introduced by the glycine residue. Although these mutants could bind GTP and GDP with high affinity (Figure 2D and data not shown), GTPase activity was not restored in either of these mutants (Figure 2E). This result strongly suggests that the conservation of a tryptophan (Trp) at position 73 is essential for the intrinsic GTP hydrolysis activity of the Rab27a protein, independently of nucleotide binding.

\section{Mutations at the Trp73 residue of Rab27a, a component of the invariant hydrophobic triad, impair effector binding}

A most interesting observation was that the spontaneous mutant Trp73Gly, which was observed in 2 patients with Griscelli syndrome, precisely affected one residue of the invariant hydrophobic triad suspected of imposing critical conformational constraints on the active form of the Rab protein, thus increasing effector-binding specificity. We have thus evaluated the ability of this mutant to interact with one of its recently described effectors, melanophilin. The specific interaction of the SHD domain of Mlph, with WT Rab27a or its mutants, was investigated in vivo using a Myc-tagged SHD domain of Mlph (Myc-SHD) and FLAG-tagged Rabs, which were coexpressed in $293 \mathrm{~T}$ cells. As expected, Myc-SHD protein was specifically found coimmunoprecipitated with the FLAGRab27a protein either in its wild-type form or Gln78Leu form, whereas the inactive GDP-bound form Thr23Asn was not (Figure 3A). Although the Trp73Gly mutant of Rab27a exhibits both the same GTP-binding activity and intrinsic GTPase deficiency as the constitutively active form Gln78Leu, the association of Trp73Gly construct with Myc-SHD could not be detected (Figure 3B). Similarly, 2 other mutants at position 73 (Trp73Leu and Trp73Ser) shown to efficiently bind GTP (Figure 1D) were unable to associate with the SHD domain of Mlph (Figure 3B). The same results were observed by immunoprecipitation with anti-Flag antibody or anti-Myc antibody (data not shown).
A WT Thr23Asn Gln78Leu

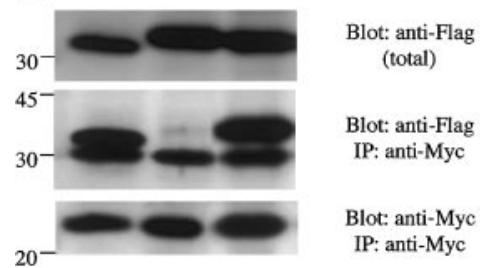

B Gln78Leu Trp73Gly Trp73Leu Trp73Ser

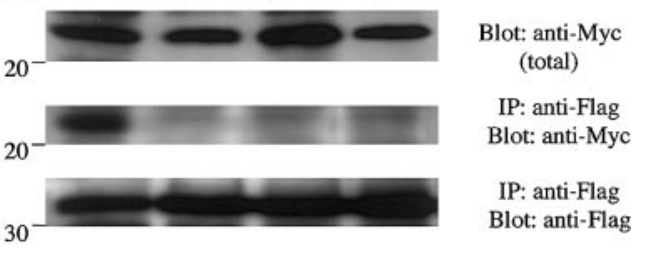

Figure 3. Interaction of the SHD domain of Mlph with Rab27a WT and mutant proteins. pFlag-CMV4-Rab27a-WT or -mutants and pMyc-cDNA3.1-SHD-Mlph were cotransfected into 293T cells and their association was analyzed by immunoprecipitation with anti-Myc antibody one day following transfection. (A) Coimmunoprecipitated Flag-Rab27a-WT or-mutant detected by anti-Flag tag antibody (IP: anti-Myc; Blot: anti-Flag). The size difference observed between WT and mutant Rab27a proteins results from the different restriction sites used for their respective cloning. The same blot was then stripped and reprobed with anti-Myc antibody to ensure that the same amounts of pMyc-SHD-Mlph proteins had been loaded (IP: anti-Myc; Blot: anti-Myc). The top panel indicates the total expressed Flag-Rab27a (1:100 volume of the reaction mixture) used for immunoprecipitation. The positions of the markers $\left(\times 10^{-3}\right)$ are shown on the left. (B) Coimmunoprecipitated pMyc-SHD-Mlph detected by anti-Myc tag antibody (IP: anti-Flag; Blot: anti-Myc). The same blot was then stripped and reprobed with anti-Flag antibody to ensure that the same amounts of Flag-Rab27a-WT or -mutant proteins had been loaded (IP: anti-Flag; Blot: anti-Flag). The top panel indicates the total expressed pMyc-SHD-Mlph (1:100 volume of the reaction mixture) used for immunoprecipitation. 
These results highlight the specific requirement of the conserved residue at position 73 for melanophilin binding, even with a GTP-bound state of the Rab27a protein. These data strongly support the contribution of a proper positioning of the invariant hydrophobic triad in effector interaction.

\section{Overexpression of Rab27a Trp73 mutants does not modify melanosome distribution}

To address the function of the Trp73 Rab27a mutants, the effect of their overexpression on melanosome distribution in melanocytes was analyzed. Transient overexpression of Trp73Gly in melan-a, a wild-type mouse melanocyte cell line, did not significantly affect the localization of the pigment granules, which were evenly distributed throughout the cytoplasm (Figure 4C-D) as observed in control enhanced GFP (EGFP)-transfected cells (Figure 4A-B). Similarly, Trp73Leu and Trp73Ser overexpression as well as Thr23Asn mutant overexpression in melan-a did not significantly modify melanososome distribution (not shown). In contrast, overexpression of the constitutively active Gln78Leu Rab27a mutant resulted in dramatic redistribution of pigment granules, which, in more than $50 \%$ of the transfected cells, were clustered in the perinuclear region (Figure 4E-F). A dominant-negative effect was also mediated by the overexpression in this cell line of the SHD domain of the Mlph able to interact with Rab27a (Figure 4G-H). Mlph truncated from its $\mathrm{C}$ terminal part cannot target myosin Va to melanosome, thus affecting melanosome mobility. Similar effects on granule positioning were observed for these various mutants used either as nontagged protein (as shown in Figure 4), whose expression was assessed by Western blot (data not shown), or tagged with an EGFP or Flag protein (not shown). Intracellular localization analysis of the transfected constructs fused with EGFP showed that the mutants' constructs were mostly detected in the cytosol rather than associated with the membrane fraction (not shown). Together, these results suggest that in these cells, because of the inability of Trp73 mutants as well as Thr23Asn mutant to bind to endogenous Rab27a effector(s), these mutants do not interfere further with the endogenous Rab27a function. In contrast, the redistribution of melanosomes observed in Rab27a Gln78Leutransfected cells may result from the rerouting and stable binding of the total pool of endogenous Rab27a effector(s) by this active mutant highly expressed in the cytosol. In this situation, the endogenous Rab27a protein is likely deprived of its effector(s) association, essential for the capture and peripheral movement of melanosomes. However, a dominant-negative effect of Rab27a Gln78Leu mutants has not been observed in previously reported studies. ${ }^{28,29}$ To better analyze the mechanism of the dominantnegative effect observed with the Gln78Leu mutant construct in our study, we investigated whether a normal phenotype could be rescued in melan-a cells cotransfected with both the SHD domain of Mlph and the Rab27a Gln78Leu mutant. The normal distribution of melanosomes observed in this situation (Figure 4I-J) strongly supports our hypothesis of a dominant-negative effect of Gln78Leu through endogenous effector capture. In addition, the absence of Trp73 mutants' effect in the same situation indirectly demonstrates in vivo the requirement of the Trp73 residue of Rab27a in specific melanophilin-effector binding.

\section{Overexpression of GIn78Leu, but not of Trp73Gly inhibits cytotoxic granule exocytosis}

We have previously shown that Rab27a mutations in patients with Griscelli syndrome affect cytotoxic granule exocytosis, a finding
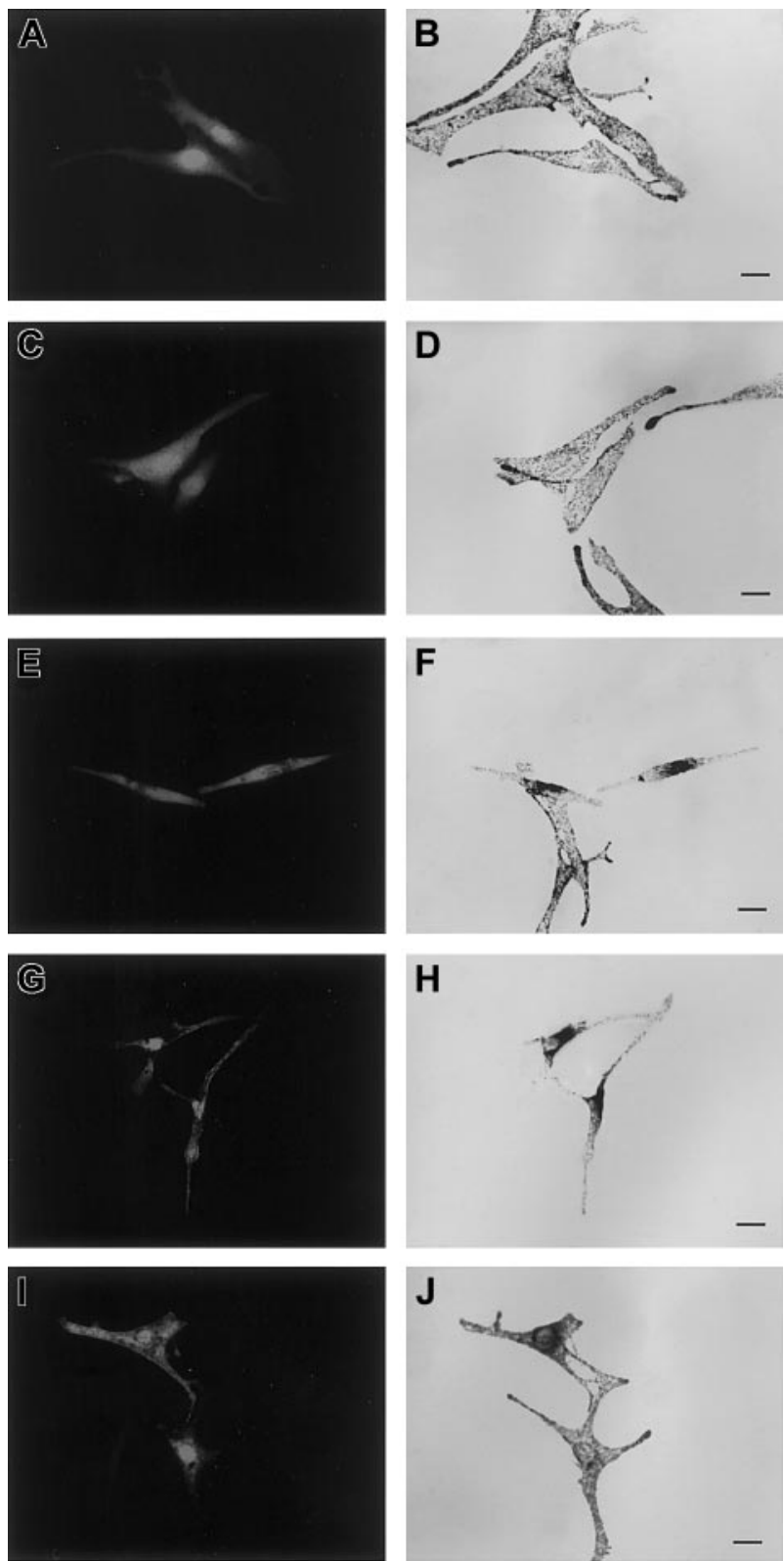

Figure 4. Overexpression of GIn78Leu but not of Trp73Gly Rab27a mutants interferes with melanosome transport, a phenotype rescued by SHD-MIph overexpression. Melan-a cells were transfected with plasmids encoding pEGFP-C1 allowing soluble GFP expression (A), or cotransfected with pEGFP-C1 and pcDNA3 encoding Trp73Gly (C), GIn78Leu (E), SHD-Mlph (G), or GIn78Leu and SHD-Mlph (I) proteins. Panels $\mathrm{B}, \mathrm{D}, \mathrm{F}, \mathrm{H}$, and $\mathrm{J}$ are images of transmitted light showing the distribution of melanosomes. Bars indicate $20 \mu \mathrm{m}$.

further confirmed in the ashen murine model. ${ }^{10,11}$ Granule exocytosis was measured by analyzing the release into the supernatant of the BLT esterase (granzyme A) activity following lymphocyte TCR triggering; this was compared with the total BLT-esterase activity contained in the lymphocyte granules. ${ }^{7}$ Granule exocytosis from patients' lymphocytes with Trp73Gly transition was undetectable, whereas in the same conditions, $50 \%$ of the granule content of control lymphocytes was released (Figure 5A). A dominant effect associated with overexpression of the Rab27a mutants was similarly assessed on granule content release from an $\mathrm{H}$ saimiritransformed cytotoxic $\mathrm{CD}^{+} \mathrm{T}$-cell line. Transient overexpression of EGFP/Rab27a-Trp73Gly construct did not significantly modify enzyme release when compared with wild-type construct effect 

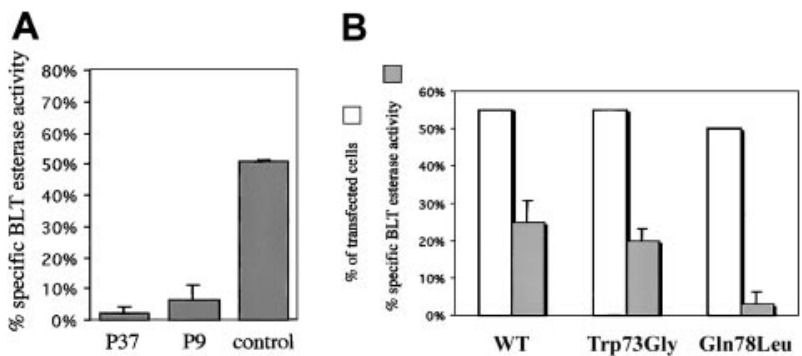

Figure 5. Effect of Rab27a mutants on granule exocytosis of cytotoxic cells. (A) Release of cytotoxic granules BLT esterase activity from the activated T cells of a control or from patients 9 (P9) and 37 (P37). Following 4-hour cytotoxic cell incubation in the presence of CD3-coated plates, cell supernatants were collected and assayed using an enzyme-linked immunosorbent assay (ELISA) for serine esterase. The data represent the mean percentage and SD of specific release (test/total release) from triplicate samples. (B) Effect of overexpression of Rab27a mutants on the cytotoxic granule exocytosis of $\mathrm{H}$ saimiri $\mathrm{CD}^{+} \mathrm{T}$-cell line. Following overexpression of Rab27a cDNAs, granule exocytosis was measured in the same conditions as in panel A (䍜). Transfection efficiency for each construct was assessed by FACScan analysis of EGFP expression ( $\square$ ). The results shown are representative of 2 independent experiments with similar results.

(Figure 5B). In contrast, overexpression of the constitutively active mutant Gln78Leu resulted in a significant inhibition of cytotoxic granule exocytosis (Figure 5B). Thus, as observed in melanocytes overexpressing the mutant constructs, Gln78Leu, but not the Trp73Gly construct, interferes with the endogenous Rab27a intracellular pathway in lymphocytes. These results strongly suggest that in both cell types, Rab27a-specific effectors' binding requires a spatial conformation of the triad, which is affected when Trp73 is mutated.

\section{Discussion}

We herein report the molecular and functional analysis of 3 missense mutations in the Rab27a gene previously identified as resulting in the expression of Griscelli syndrome phenotype. ${ }^{7}$ The introduction of a proline in the Rab27a protein, located either in the $\beta 5$ sheet (Leu130Pro) or the $\alpha 4$ helix (Ala152Pro), interferes with guanine nucleotide-binding activity, strongly suggesting that these mutations perturb the overall structure of the protein. In contrast, the $\operatorname{Trp} \rightarrow$ Gly transition at position 73 , characterized in a third spontaneous mutant, does not alter GTP or GDP binding but reduces the basal GTPase activity of Rab27a. Residue 73 belongs to the RabF2 prototypical sequence signature of the Rab family, and is located in the $\beta 3$ sheet, at the interface between the 2 switch regions (reviewed in Pereira-Leal and Seabra ${ }^{5}$ ). The replacement of a hydrophobic aromatic residue (Trp), with a polar and tiny residue (Gly) that is devoid of lateral chain, could have introduced a higher flexibility at the switch interface, modifying the dynamics of the switch conformational change. The Trp position is conserved in all small GTPases, except in Ras, where a leucine, another hydrophobic residue, is present. However, the introduction of a leucine residue in position 73 of the Rab27a protein was inefficient to restore its intrinsic GTPase activity. Similarly, GTPase activity of Rab27a was not restored by the presence of a serine residue at this position, a residue chosen for its ability to limit the flexibility of the switch II region, potentially increased by the glycine transition. Conservation of Trp at position 73 thus appears determinant in maintaining the intrinsic GTP hydrolysis activity of Rab27a. Similarly to the Gln78Leu mutation, the Trp73Gly spontaneous mutant displays a constitutively active profile in terms of in vitro nucleotide-associated characteristics. However, these 2 mutants dramatically differ in their ability to interact with the SHD domain of the melanophilin, a recently identified effector of Rab27a. ${ }^{13,15-17}$ The Trp73Gly mutant was unable to associate with its specific effector, while the Gln78Leu mutant displayed a high affinity for it, demonstrating that Trp73 residue has a major role in effector binding. Lack of GTPase activity of Trp73Gly/Trp73Leu and Trp73Ser argue against a "native" conformation of the switch regions when other residues replace Trp. The configuration state that allows hydrolysis is probably not well populated. This differs from Gln78Leu in which defective hydrolysis is due to a lack of stabilization of a water molecule. ${ }^{30}$ Trp 73 is one of the 3 hydrophobic conserved residues at the switch interface, previously shown in Rab3A to participate in Rab3A-rabphilin3A interaction. In the Rab3/rabphilin complex, the $3 \mathrm{Rab} 3 \mathrm{~A}$ residues of the triad (ie, Phe59, Trp76, and Tyr91) are involved in a few van der Waals interactions with an apolar side of the first long helix of rabphilin (residues Arg60 and Ala61 for Phe59; residue Ala61 for Trp76; and residue Ile58 for Tyr91). Since melanophilin and rabphilin-3A are highly similar for their Rab-binding domain (among the 25 residues involved in interactions with Rab3 in the crystal structure, 9 residues are identical and 6 are conservative changes), it is likely that the overall interface between each Rab-effector complex is similar. This invariant hydrophobic triad is thought to increase effector recognition specificity through the spatial conformation adopted by these residues in the active conformation of the protein. None of the mutants introduced at position 73 was able to restore this function. Lack of Trp may reorganize the position mostly adopted by the other 2 side chains of the triad and, by changing the interface, would reduce dramatically the binding of Mlph. These data demonstrate a second determinant role of this amino acid in effector association, thereby further supporting its contribution in specific effectors' recognition.

Functional difference between these Trp73 and Gln78 mutants characteristics was also evidenced by their distinct effect on melanosome relocalization in melan-a cells, as well as in cytotoxic granule release. Gln78Leu mutant was able to exert only a dominant-negative effect, leading to perinuclear clustering of melanososomes in melanocytes and inhibition of cytotoxic granule exocytosis in lymphocytes. This dominant-negative effect resulted from the sequestration of an upstream activator, since it was inhibited by the coexpression of melanophilin in melanocytes. In accordance with this observation, all other mutants, including Trp73Gly, unable to coimmunoprecipitate with melanophilin did not modify melanosome transport. It is worth noting that a dominant-negative effect of the inactive Thr23Asn mutant has been reported (in $13 \%{ }^{28}$ to $80 \%^{29}$ of the melanocytes), whereas the active Gln78Leu construct had no effect. In our study, mutants have the same effect in melanocytes and in cytotoxic cells; this is true with either tagged or untagged expressed Rab27a constructs of these mutants, thus strengthening the specificity of the observed effects. Such variations may result from differences in Rab27a mutants' expression level and/or in differential membrane association of the Rab27a mutants' constructs among experiments.

Although Mlph was shown to be expressed in melanocytes, its presence is undetectable in cytotoxic cells, and accordingly, mutation in Mlph, which characterized leaden mice, does not affect cytotoxic activity. ${ }^{16}$ It was thus hypothesized either that another member of the Mlph family specifically interacts with Rab27a for inducing cytotoxic granule exocytosis or that structurally unrelated effectors regulate this function in cytotoxic cells. The observation that Gln78Leu but not Trp73Gly exerts a dominant-negative effect 
on cytotoxic granule exocytosis is in favor of the former hypothesis, since interaction of Rab27a with a specific effector in cytotoxic cells requires fine structural constraints indistinguishable from the one enabling Rab27a-Mlph interaction in melanocytes. Future experiments should thus identify the specific effector(s) of Rab27a in cytotoxic cells, a functionally critical molecule in triggering cell cytotoxicity.

All the patients with Griscelli syndrome due to Rab27a mutation developed an HS consecutive to the functional impairment of the secretory cytotoxic pathway. Failure to kill antigenpresenting cells by effector cytotoxic cells, can favor a sustained immune response, and therefore the persistence of overwhelming activated $\mathrm{T}$ lymphocytes. These $\mathrm{T}$ lymphocytes will lead to macrophage activation, secretion of inflammatory cytokines and their subsequent deleterious effect in infiltrated organs. ${ }^{8}$ Although comparison of disease severity among patients from various geographic and medical contexts is a difficult task, it is interesting to note that patients with either Ala152Pro or Trp73Gly missense mutations had a later onset of HS than patients with null mutation. This suggests a possible in vivo residual activity of these mutated Rab proteins. Further analysis of mutated Rab27a proteins could contribute to both a better assessment of the structure/function relationship of Rab27a and of in vivo immunologic comparison.

\section{Acknowledgments}

We thank Dorothy Bennett, Helmut Fickenscher, and Pier Giorgio Natali for providing us with melan-a cells, H saimiri C3 T cells, and MNT1 cell line, respectively.

\section{References}

1. Milburn MV, Tong L, deVos AM, et al. Molecular switch for signal transduction: structural differences between active and inactive forms of protooncogenic ras proteins. Science. 1990;247:939-945.

2. Chavrier P, Goud B. The role of ARF and Rab GTPases in membrane transport. Curr Opin Cell Biol. 1999;11:466-475.

3. Takai Y, Sasaki T, Matozaki T. Small GTP-binding proteins. Physiol Rev. 2001;81:153-208.

4. Martinez O, Goud B. Rab proteins. Biochim Biophys Acta. 1998;1404:101-112.

5. Pereira-Leal JB, Seabra MC. The mammalian Rab family of small GTPases: definition of family and subfamily sequence motifs suggests a mechanism for functional specificity in the Ras superfamily. J Mol Biol. 2000;301:1077-1087

6. Seabra MC, Mules EH, Hume AN. Rab GTPases, intracellular traffic and disease. Trends Mol Med. 2002;8:23-30.

7. Ménasché G, Pastural E, Feldmann J, et al. Mutations in RAB27A cause Griscelli syndrome associated with hemophagocytic syndrome. Nat Genet. 2000;25:173-176

8. de Saint Basile G, Fischer A. The role of cytotoxicity in lymphocyte homeostasis. Curr Opin Immu nol. 2001;13:549-554.

9. Wilson SM, Yip R, Swing DA, et al. A mutation in Rab27a causes the vesicle transport defects observed in ashen mice. Proc Natl Acad Sci U S A. 2000;97:7933-7938

10. Haddad EK, Wu X, Hammer JA, Henkart PA. Defective granule exocytosis in rab27a-deficient lymphocytes from ashen mice. J Cell Biol. 2001; 152:835-842.

11. Stinchcombe JC, Barral DC, Mules EH, et al. Rab27a is required for regulated secretion in cytotoxic T lymphocytes. J Cell Biol. 2001;152:825834.

12. Matesic LE, Yip R, Reuss AE, et al. Mutations in
Mlph, encoding a member of the Rab effector family, cause the melanosome transport defects observed in leaden mice. Proc Natl Acad Sci U S A. 2001;98:10238-10243.

13. Fukuda M, Kuroda TS, Mikoshiba K. Slac2-a/ melanophilin, the missing link between Rab27 and myosin Va: implications of a tripartite protein complex for melanosome transport. J Biol Chem. 2002;277:12432-12436.

14. Kuroda TS, Fukuda M, Ariga H, Mikoshiba K. The Slp homology domain of synaptotagmin-like proteins 1-4 and Slac2 functions as a novel Rab27A binding domain. J Biol Chem. 2002;277:92129218.

15. Nagashima K, Torii S, Yi Z, et al. Melanophilin directly links Rab27a and myosin Va through its distinct coiled-coil regions. FEBS Lett. 2002;517 233-238.

16. Hume AN, Collinson LM, Hopkins CR, et al. The leaden gene product is required with Rab27a to recruit myosin Va to melanosomes in melanocytes. Traffic. 2002;3:193-202.

17. Wu XS, Rao K, Zhang $\mathrm{H}$, et al. Identification of an organelle receptor for myosin-Va. Nat Cell Biol. 2002;4:271-278.

18. Ostermeier C, Brunger AT. Structural basis of Rab effector specificity: crystal structure of the small G protein Rab3A complexed with the effector domain of rabphilin-3A. Cell. 1999;96:363-374.

19. Merithew E, Hatherly S, Dumas JJ, Lawe DC, Heller-Harrison R, Lambright DG. Structural plasticity of an invariant hydrophobic triad in the switch regions of Rab GTPases is a determinant of effector recognition. J Biol Chem. 2001;276: 13982-13988.

20. Sanal O, Ersoy F, Tezcan I, et al. Griscelli disease: genotype-phenotype correlation in an array of clinical heterogeneity. J Clin Immunol. 2002;22: 237-243.

21. Griscelli C, Durandy A, Guy-Grand D, Daguillard F, Herzog C, Prunieras M. A syndrome associat- ing partial albinism and immunodeficiency. Am J Med. 1978;65:691-702.

22. Echard A, Jollivet F, Martinez $\mathrm{O}$, et al. Interaction of a Golgi-associated kinesin-like protein with Rab6. Science. 1998;279:580-585.

23. Seraphin B, Kandels-Lewis S, Leong PM, Miller $\mathrm{JH}$, Calos MP, Fleckenstein B. An efficient PCR mutagenesis strategy without gel purification step that is amenable to automation. Nucleic Acids Res. 1996;24:3276-3277.

24. Hoffenberg S, Sanford JC, Liu S, et al. Biochemical and functional characterization of a recombinant GTPase, Rab5, and two of its mutants. J Biol Chem. 1995;270:5048-5056.

25. DuBridge RB, Tang P, Hsia HC, et al. Analysis of mutation in human cells by using an Epstein-Barr virus shuttle system. Mol Cell Biol. 1987;7:379387

26. Fickenscher $\mathrm{H}$, Biesinger $\mathrm{B}$, Knappe A, Wittmann $\mathrm{S}$, Fleckenstein $\mathrm{B}$. Regulation of the herpesvirus saimiri oncogene stpC, similar to that of T-cell activation genes, in growth-transformed human $\mathrm{T}$ lymphocytes. J Virol. 1996;70:6012-6019.

27. Davidson D, Cloutier JF, Gregorieff A, Veillette A Inhibitory tyrosine protein kinase p50csk is associated with protein-tyrosine phosphatase PTPPEST in hemopoietic and non-hemopoietic cells. J Biol Chem. 1997;272:23455-23462.

28. Hume AN, Collinson LM, Rapak A, Gomes AQ, Hopkins CR, Seabra MC. Rab27a regulates the peripheral distribution of melanosomes in melanocytes. J Cell Biol. 2001;152:795-808.

29. Wu X, Rao K, Bowers MB, Copeland NG, Jenkins NA, Hammer JA. Rab27a enables myosin Vadependent melanosome capture by recruiting the myosin to the organelle. J Cell Sci. 2001;114: 1091-1100.

30. Frech M, Darden TA, Pedersen LG, et al. Role of glutamine- 61 in the hydrolysis of GTP by $\mathrm{p} 21 \mathrm{H}$ ras: an experimental and theoretical study. Biochemistry. 1994;33:3237-3244. 